PDFlib PLOP: PDF Linearization, Optimization, Protection

Page inserted by evaluation version www.pdflib.com - sales@pdflib.com 


\title{
Power Spectrum Analysis of Large Baseline Redshift Surveys*
}

\author{
Hume A. Feldman \\ Physics Department \\ University of Michigan \\ Ann Arbor, MI 48109
}

Given a set of redshifts of galaxies with known angular and luminousity criteria, our goal is to construct a descriptive statistic that measures the power spectrum of the underlying density fluctuation field. We assume that the fluctuation field is some homogeneous and statistically isotropic random process[1]. The present study follows the program laid down by Peebles in his pioneering series of papers[2] for statistical analysis of galaxy catalogues via low order correlation functions.

We make the usual assumption that the galaxies form a poisson sample[3] of the density field $1+f(\mathbf{r})=\rho(\mathbf{r}) / \bar{\rho}$ :

$$
P(\text { vol element } \delta V \text { contains a galaxy })=\delta V \bar{n}(\mathbf{r})(1+f(\mathbf{r}))
$$

where $\bar{n}(\mathbf{r})$ is the expected mean space density of galaxies given the angular and luminosity selection criteria, and we wish to estimate the power spectrum

$$
P(k)=P(\mathbf{k}) \equiv \int d^{3} r \xi(\mathbf{r}) e^{i \mathbf{k} \cdot \mathbf{r}}
$$

where $\xi(\mathbf{r})=\xi(r)=\left\langle f\left(\mathbf{r}^{\prime}\right) f\left(\mathbf{r}^{\prime}+\mathbf{r}\right)\right\rangle$ is the 2-point correlation function.

Our approach is to take the fourier transform of the real galaxies minus the transform of a synthetic catalogue with the same angular and radial selection function as the real galaxies but otherwise without structure. We also incorporate a weight function $w(\mathbf{r})$ which will be adjusted to optimize the performance. We define the weighted galaxy fluctuation field, to be

$$
F(\mathbf{r}) \equiv \frac{w(\mathbf{r})\left(n_{g}(\mathbf{r})-\alpha n_{s}(\mathbf{r})\right)}{\left(\int d^{3} r \bar{n}^{2}(\mathbf{r}) w^{2}(\mathbf{r})\right)^{1 / 2}}
$$

where $n_{g}(\mathbf{r})=\sum_{i} \delta\left(\mathbf{r}-\mathbf{r}_{i}\right)$ with $\mathbf{r}_{i}$ being the location of the ith galaxy and similarly for the synthetic catalogue which has number density $1 / \alpha$ times that of the real catalogue.

Taking the fourier transform of $F(\mathbf{r})$, squaring it and taking the expectation value we find:

$$
\left\langle|F(\mathbf{k})|^{2}\right\rangle=\frac{\int d^{3} r \int d^{3} r^{\prime} w(\mathbf{r}) w\left(\mathbf{r}^{\prime}\right)\left\langle\left[n_{g}(\mathbf{r})-\alpha n_{s}(\mathbf{r})\right]\left[n_{g}\left(\mathbf{r}^{\prime}\right)-\alpha n_{s}\left(\mathbf{r}^{\prime}\right)\right]\right\rangle e^{i \mathbf{k} \cdot\left(\mathbf{r}-\mathbf{r}^{\prime}\right)}}{\int d^{3} r \bar{n}^{2}(\mathbf{r}) w^{2}(\mathbf{r})}
$$

This work was supported in part by the National Science Foundation Grant NSF-PHY-92-96020 
With the model of equation 1 , the two point functions of $n_{g}, n_{s}$ are

$$
\begin{aligned}
& \left\langle n_{g}(\mathbf{r}) n_{g}\left(\mathbf{r}^{\prime}\right)\right\rangle=\bar{n}(\mathbf{r}) \bar{n}\left(\mathbf{r}^{\prime}\right)\left(1+\xi\left(\mathbf{r}-\mathbf{r}^{\prime}\right)\right)+\bar{n}(\mathbf{r}) \delta\left(\mathbf{r}-\mathbf{r}^{\prime}\right) \\
& \left\langle n_{s}(\mathbf{r}) n_{s}\left(\mathbf{r}^{\prime}\right)\right\rangle=\alpha^{-2} \bar{n}(\mathbf{r}) \bar{n}\left(\mathbf{r}^{\prime}\right)+\alpha^{-1} \bar{n}(\mathbf{r}) \delta\left(\mathbf{r}-\mathbf{r}^{\prime}\right) \\
& \left\langle n_{g}(\mathbf{r}) n_{s}\left(\mathbf{r}^{\prime}\right)\right\rangle=\alpha^{-1} \bar{n}(\mathbf{r}) \bar{n}\left(\mathbf{r}^{\prime}\right)
\end{aligned}
$$

so

$$
\left\langle|F(\mathbf{k})|^{2}\right\rangle=\int \frac{d^{3} k^{\prime}}{(2 \pi)^{3}} P\left(\mathbf{k}^{\prime}\right)\left|G\left(\mathbf{k}-\mathbf{k}^{\prime}\right)\right|^{2}+\mathrm{P}_{\text {shot }}
$$

where

$$
G(\mathbf{k}) \equiv \frac{\int d^{3} r \bar{n}(\mathbf{r}) w(\mathbf{r}) e^{i \mathbf{k} \cdot \mathbf{r}}}{\left(\int d^{3} r \bar{n}^{2}(\mathbf{r}) w^{2}(\mathbf{r})\right)^{1 / 2}}
$$

and

$$
\mathrm{P}_{\text {shot }} \equiv(1+\alpha) \frac{\int d^{3} r \bar{n}(\mathbf{r}) w^{2}(\mathbf{r})}{\int d^{3} r \bar{n}^{2}(\mathbf{r}) w^{2}(\mathbf{r})} .
$$

For a large baseline survey $G(\mathbf{k})$ is a rather compact function with width $\sim 1 / D$, where $D$ characterizes the depth of the survey. Provided we restrict attention to $|\mathbf{k}| \gg 1 / D$, which is really just the requirement that we have a 'fair sample', and provided $P(\mathbf{k})$ is locally smooth on the same scale, then

$$
\left\langle|F(\mathbf{k})|^{2}\right\rangle \simeq P(\mathbf{k})+P_{\text {shot }},
$$

so the raw power spectrum $|F(\mathbf{k})|^{2}$ is the true power spectrum plus the constant shot noise component and our estimator is

$$
\hat{P}(\mathbf{k})=|F(\mathbf{k})|^{2}-P_{\text {shot }},
$$

finally we average over a shell in $k$-space:

$$
\hat{P}(k) \equiv \frac{1}{V_{k}} \int_{V_{k}} d^{3} k^{\prime} \hat{P}\left(\mathbf{k}^{\prime}\right)
$$

where $V_{k}$ is the volume of the shell.

Equations (3), (9-11) provide our operational definition of $\hat{P}(k)$. To use these we must specify the weight function $w(\mathbf{r})$ which so far has been arbitrary, and we must choose some sampling grid in $k$-space. In order to set these wisely - and also to put error bars on our estimate of the power - we need to understand the statistical fluctuations in $\hat{P}(\mathbf{k})$.

From equation (11) the mean square fluctuation in $\hat{P}(k)$ is

$$
\sigma_{P}^{2} \equiv\left\langle(\hat{P}(k)-P(k))^{2}\right\rangle=\frac{1}{V_{k}^{2}} \int_{V_{k}} d^{3} k \int_{V_{k}} d^{3} k^{\prime}\left\langle\delta \hat{P}(\mathbf{k}) \delta \hat{P}\left(\mathbf{k}^{\prime}\right)\right\rangle .
$$


An interesting model for the two point function of $\delta \hat{P}(\mathbf{k})$ is to assume that the coefficients $F(\mathbf{k})$ are gaussian distributed, in which case $\left\langle\delta \hat{P}(\mathbf{k}) \delta \hat{P}\left(\mathbf{k}^{\prime}\right)\right\rangle=$ $\left|\left\langle F(\mathbf{k}) F^{*}\left(\mathbf{k}^{\prime}\right)\right\rangle\right|^{2}$. A generalisation of the steps leading to (6) gives us

$$
\left\langle F(\mathbf{k}) F^{*}\left(\mathbf{k}^{\prime}\right)\right\rangle=\int \frac{d^{3} k^{\prime \prime}}{(2 \pi)^{3}} P\left(\mathbf{k}^{\prime \prime}\right) G\left(\mathbf{k}-\mathbf{k}^{\prime \prime}\right) G^{*}\left(\mathbf{k}^{\prime}-\mathbf{k}^{\prime \prime}\right)+S\left(\mathbf{k}^{\prime}-\mathbf{k}\right),
$$

where we have defined

$$
S(\mathbf{k}) \equiv \frac{(1+\alpha) \int d^{3} r \bar{n}(\mathbf{r}) w^{2}(\mathbf{r}) e^{i \mathbf{k} \cdot \mathbf{r}}}{\int d^{3} r \bar{n}^{2}(\mathbf{r}) w^{2}(\mathbf{r})}
$$

and, in the same approximation that led to equation (9) we obtain

$$
\left\langle F(\mathbf{k}) F^{*}(\mathbf{k}+\delta \mathbf{k})\right\rangle \simeq P(\mathbf{k}) Q(\delta \mathbf{k})+S(\delta \mathbf{k})
$$

where

and therefore

$$
Q(\mathbf{k}) \equiv \frac{\int d^{3} r \bar{n}^{2}(\mathbf{r}) w^{2}(\mathbf{r}) e^{i \mathbf{k} \cdot \mathbf{r}}}{\int d^{3} r \bar{n}^{2}(\mathbf{r}) w^{2}(\mathbf{r})}
$$

$$
\left\langle\delta \hat{P}(\mathbf{k}) \delta \hat{P}\left(\mathbf{k}^{\prime}\right)\right\rangle=|P(\mathbf{k}) Q(\delta \mathbf{k})+S(\delta \mathbf{k})|^{2} .
$$

If the shell we average over in equation (11) has a width which is large compared to the coherence length then the double integral in (12) reduces to

$$
\sigma_{P}^{2}(k) \simeq \frac{1}{V_{k}} \int d^{3} k^{\prime}\left|P(k) Q\left(\mathbf{k}^{\prime}\right)+S\left(\mathbf{k}^{\prime}\right)\right|^{2},
$$

so, with the definition of $Q(\mathbf{k})$ and $S(\mathbf{k})$ and using Parseval's theorem, the fractional variance in the power is

$$
\sigma_{P}^{2}(k) / P(k)^{2}=(2 \pi)^{3} \int d^{3} r \bar{n}^{4} w^{4}(1+1 / \bar{n} P(k))^{2} / V_{k}\left[\int d^{3} r \bar{n}^{2} w^{2}\right]^{2} .
$$

We seek $w(\mathbf{r})$ which minimises this. Writing $w(\mathbf{r})=w_{0}(\mathbf{r})+\delta w(\mathbf{r})$ and requiring that $\sigma_{P}^{2}(k)$ be stationary with respect to arbitrary variations $\delta w(\mathbf{r})$ we obtain

$$
\frac{\int d^{3} r \bar{n}^{4} w_{0}^{3}\left(\frac{1+\bar{n} P}{\bar{n} P}\right)^{2} \delta w(\mathbf{r})}{\int d^{3} r \bar{n}^{4} w_{0}^{4}\left(\frac{1+\bar{n} P}{\bar{n} P}\right)^{2}}=\frac{\int d^{3} r \bar{n}^{2} w_{0} \delta w(\mathbf{r})}{\int d^{3} r \bar{n}^{2} w_{0}^{2}}
$$

and it is easy to see by direct substitution that this is satisfied if we take

$$
w_{0}(\mathbf{r})=[1+\bar{n}(\mathbf{r}) P(k)]^{-1} .
$$

This is the optimal weighting (under the assumption that the fluctuations are gaussian).

\section{References}

[1] Feldman, H.A., Kaiser, N. \& Peacock, J., 1993 in preparation.

[2] Peebles, P.J.E. \& Hauser, G.M., 1974 ApJ Suppl. 28 19; Yu, J.T. \& Peebles, P.J.E., 1969 ApJ 158103.

[3] Peebles, P.J.E. 1980, The Large Scale Structure of the Universe, Princeton U. Press. 